\title{
CROP YIELD MAP DEVELOPMENT BASED ON SATELLITE AND UAV DATA
}

\author{
Irina Botvich $^{1,2^{*}}$, Anatoly Shevyrnogov ${ }^{1}$, and Dmitriy Emelyanov ${ }^{1}$ \\ ${ }^{1}$ Institute of Biophysics SB RAS,660036 Krasnoyarsk, Russia \\ ${ }^{2}$ Siberian Federal University, 660041 Krasnoyarsk, Russia
}

\begin{abstract}
Agriculture is one of the promising areas for the introduction of remote sensing technologies. With its help, you can timely receive a wide range of dynamic information about the conditions for the crops growth and development and make the necessary adjustments to obtain the planned result. The paper presents the results of early forecasting of spring wheat yields based on Dove (PlanetScope) satellite data from Planet Labs and DJI P4 Multispectral unmanned data. The maps of the yield of spring wheat were constructed using satellite and unmanned data with a spatial resolution of 3 $\mathrm{m}$ and $5 \mathrm{~cm}$, respectively. A statistical assessment of the inhomogeneity of the spectral optical characteristics of agricultural crops has been carried out. The degree of correlation dependence between the value of the integral of the index (NDVI, VARI, MSAVI2, ClGreen) and the yield in different periods of the growing season has been determined.
\end{abstract}

Keywords: precision farming, yield, growing season, spring wheat, PlanetScope, DJI P4 Multispectral

\section{Introduction}

The widespread introduction of energy-saving technologies for the cultivation of agricultural crops is aimed at reducing material and technical costs in the crop production. An increase in the level of profitability of agricultural production in modern conditions is possible only on the basis of the introduction of modern energy-saving technologies for growing plants using elements of precision farming Remote sensing methods make it possible to predict the yield of field crops and, at the same time, to assess the crops state (weediness, plant density, damage by pests and diseases, the state of crops after overwintering, etc.) and, ultimately, to manage production process. The purpose of this study is to develop a precision farming method for early forecasting of grain yield based on remote sensing data (satellite and unmanned aerial vehicle - UAV) and geobotanical studies.

\footnotetext{
* Corresponding author: irina.pugacheva@mail.ru
} 


\section{Objects and Methods}

The studies were carried out on the lands of the Minderlinskoye educational farm in the Sukhobuzimsky district of the Krasnoyarsk Territory during the growing seasons of 20192020. Figure 1 shows the location of the experimental field and its structure. The experimental field consists of 5 strips. Their average length is 650 meters. The direction of the strips is from west to east, the width of each strip is $20 \mathrm{~m}$. Fertilization was carried out along the northern part of each strip (10 m wide), the southern part remained without fertilizers. Each strip is sown with a specific type of crops.

The paper presents an analysis of the data processing results on the strips sown with wheat "Novosibirskaya-15". The fields are divided into 4 test areas in accordance with four types of soil cultivation: plowing $(20-22 \mathrm{~cm})$ - a; sweep treatment $(20-22 \mathrm{~cm})-b$; surface treatment (discator by $8-10 \mathrm{~cm}$ ) - c; no tillage - $\mathrm{d}$. For the purity of the experiment, all test areas are separated from each other by protective stripes 5 meters wide. Accounting for the yield of wheat grain was carried out by a continuous method using a combine harvester in 6 replicates in 2019 and 4 replicates in 2020, for each type of soil treatment. The grain yield was calculated in centners per hectare, taking into account the conversion to $14 \%$ moisture and $100 \%$ purity.

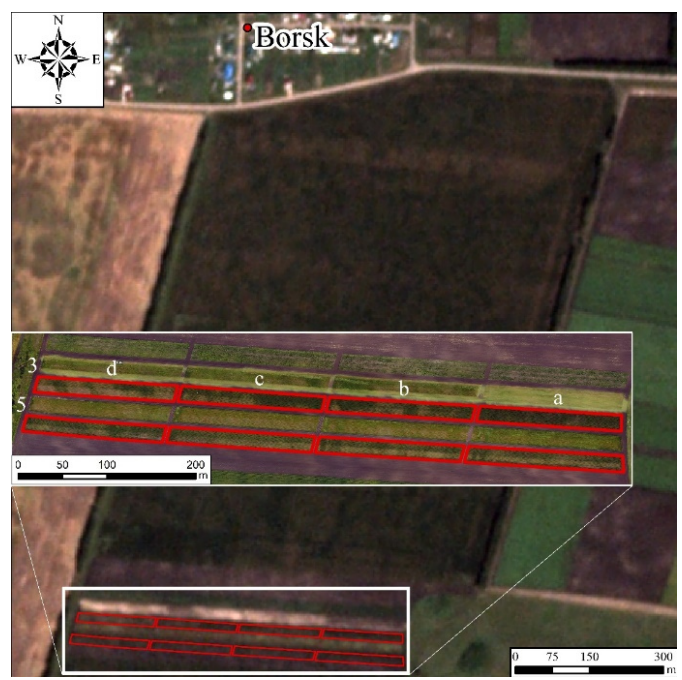

Fig. 1. Study site and its structure by types of processing and crops. Lines 3 and 5 are sown with spring wheat. Borders of different types of tillage: $\mathrm{d}, \mathrm{c}, \mathrm{b}, \mathrm{a}-$ in the direction from left to right.

The study is based on satellite data from PlanetScope (spatial resolution $3 \mathrm{~m}$ ) and UAV data from the DJI P4 Multispectral (spatial resolution $5 \mathrm{~cm}$ ). The spectral indices NDVI (Normalized Difference Vegetation Index) [1], VARI (Visible Atmospherically Resistant Index) [2], MSAVI2 (Modified Soil Adjusted Vegetation Index) [3], ClGreen (Green chlorophyll index) were calculated using satellite and unmanned spectrophotometric data. [4] (Table 1).

Table 1. Satellite and unmanned spectral channels for calculating indices:

b1 - 1 channel reflectance (455 $-515 \mathrm{~nm})$ of PlanetScope; $450 \mathrm{~nm}( \pm 16 \mathrm{~nm})$ DJI P4 Multispectral; b2 - 2 channel reflectance $(500-590 \mathrm{~nm})$ of PlanetScope; $560 \mathrm{~nm}( \pm 16 \mathrm{~nm})$ DJI P4 Multispectral; b3 - 3 channel reflectance $(590-670 \mathrm{~nm})$ of PlanetScope; $650 \mathrm{~nm}( \pm 16 \mathrm{~nm})$ DJI P4 Multispectral; 
b4 - 4 channel reflectance (780 - $860 \mathrm{~nm})$ of PlanetScope; 5 channel $840 \mathrm{~nm}( \pm 16 \mathrm{~nm})$ DJI P4 Multispectral.

\begin{tabular}{|c|c|}
\hline Index & Formula \\
\hline NDVI & $(\mathrm{b} 4-\mathrm{b} 3) /(\mathrm{b} 4+\mathrm{b} 3)$ \\
\hline VARI & $(\mathrm{b} 2-\mathrm{b} 3) /(\mathrm{b} 2+\mathrm{b} 3-\mathrm{b} 1)$ \\
\hline MSAVI2 & $\left(2 * \mathrm{~b} 4+1-\left((2 * \mathrm{~b} 4+1)^{2}-8 *(\mathrm{~b} 4-\mathrm{b} 3)\right)^{1 / 2}\right) / 2$ \\
\hline ClGreen & $(\mathrm{b} 4 / \mathrm{b} 2)-1$ \\
\hline
\end{tabular}

The calculation of the curvilinear integral of the studied indices (I) is carried out according to the formula:

$$
I=\sum_{k=1}^{n}\left(\left(\frac{V_{k}+V_{k+1}}{2}\right) \cdot\left(d_{k+1}-d_{k}\right)\right)
$$

where $V_{k}$ is the value of the vegetation index, $d_{k}$ is the time (day of survey), $n$ is the number of measurements.

\section{Results and discussion}

To determine the relationship between the integrals of the index values and the yield of wheat, the coefficient of determination $\left(\mathrm{r}^{2}\right)$ between these variables was calculated. Wheat yield was measured in 4 replicates for each type of treatments (64 plots in total). For the same areas, the values of the integrals of the indices NDVI, MSAVI2, VARI, ClGreen were determined. Analysis of the relationship between the yield and the value of the integral during the growing season of 2020 (according to satellite data) showed the presence of a more pronounced plateau. At the end of June (06/26/2020), the $\mathrm{r}^{2}$ value reaches high values, more than 0.7 for MSAVI2 and ClGreen. For NDVI values, $\mathrm{r}^{2}$ are significantly lower than 0.59 . The lowest values are obtained for the VARI 0.53 integral (fig. 2).

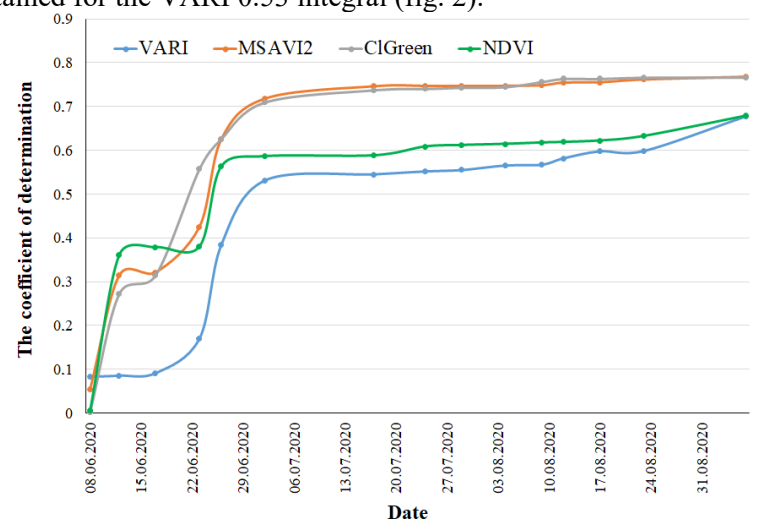

Fig. 2. Coefficients of determination between wheat yield and the value of the integral of the NDVI, MSAVI2, VARI, ClGreen indices, calculated using satellite data during the growing season of 2020.

Analysis of the relationship between the yield and the integral value during the growing season of 2020 (according to unmanned data) showed that from the beginning of July the value of $\mathrm{r}^{2}$ reaches sufficiently significant values (from 0.42 to 0.5 ) for all indices, while the 
NDVI integral has the minimum value, the maximum ClGreen and MSAVI2. The maximum values were reached by August 20 - 0.64 (ClGreen) and 0.69 MSAVI2. The maximum values of the NDVI integral are 0.71 by the first days of September.

Analysis of the relationship between the yield and the integral value during the growing season of 2019 (according to satellite data) showed that the coefficient of determination $\mathrm{r}^{2}$ has the highest values when using the NDVI integral. Since the first days of July, $\mathrm{r}^{2}$ has exceeded 0.86. The VARI integral had the lowest values during this time period. At the end of the growing season (before harvesting), all the integrals of the indices showed a high degree of relationship with yield - from 0.84 for VARI to 0.93 for NDVI, the determination values MSAVI2, ClGreen are 0.9 .

Thus, an analysis of the data obtained over two years of research on satellite and unmanned measurements allows us to conclude that the most optimal and reliable is the use of integrals of the MSAVI2 index.

Representation of information in the form of accumulated values of integrals MSAVI2 shows that, in comparison with the spatial variation of direct values of MSAVI2, a structure that is stable in time appears for all types of treatments. At the same time, there is a clear dependence of the change in the spatial structure of the MSAVI2 integrals in time (Figs. 3, 4).

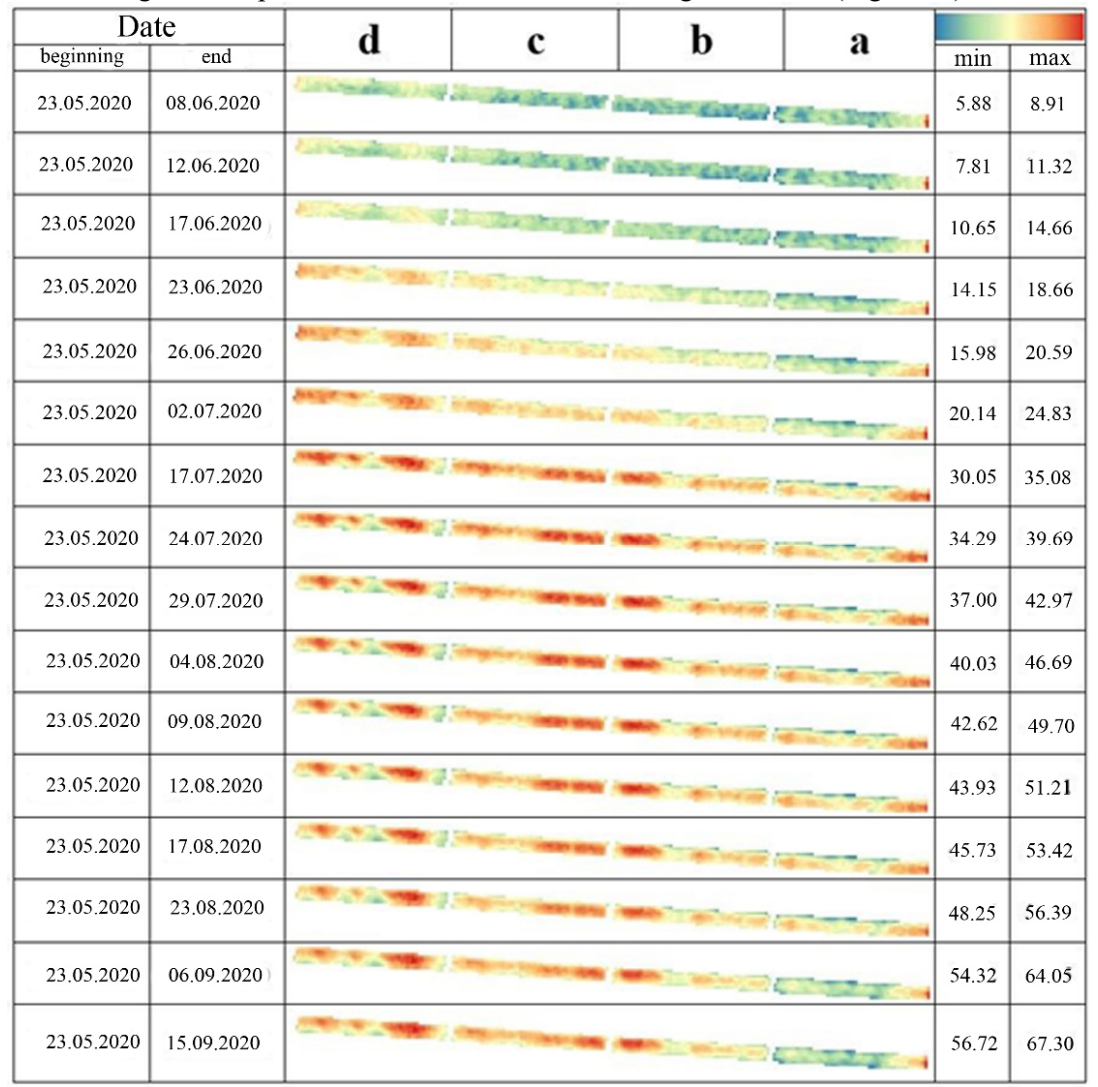

Fig. 3. Cartograms of the MSAVI2 wheat crops integral during the growing season (from May 23 to September 15, 2020) obtained from satellite data. 


\begin{tabular}{|c|c|c|c|c|c|c|}
\hline \multicolumn{2}{|c|}{ Date } & \multirow{2}{*}{\multicolumn{2}{|c|}{\begin{tabular}{l|l}
$\mathbf{d}$ & $\mathbf{C}$ \\
\end{tabular}}} & \multirow{2}{*}{\begin{tabular}{|l|l}
$\mathbf{b}$ & $\mathbf{a}$ \\
\end{tabular}} & \multirow{2}{*}{$\min$} & \multirow[b]{2}{*}{$\max$} \\
\hline beginning & end & & & & & \\
\hline 18.06 .2020 & 26.06 .2020 & \multicolumn{3}{|c|}{ 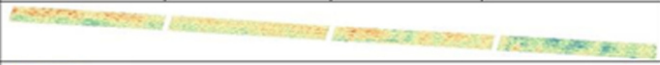 } & 0.012 & 0.103 \\
\hline 18.06 .2020 & 03.07 .2020 & \multicolumn{3}{|c|}{ 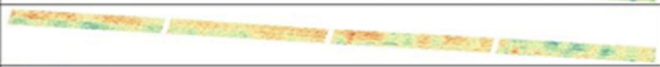 } & 0.029 & 0.198 \\
\hline 18.06 .2020 & 13.07 .2020 & \multicolumn{3}{|c|}{ 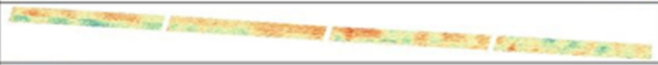 } & 0.058 & 0.336 \\
\hline 18.06 .2020 & 22.07 .2020 & \multicolumn{3}{|c|}{ 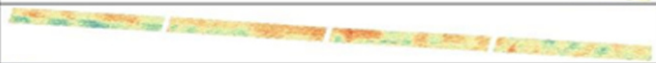 } & 0.079 & 0.464 \\
\hline 18.06 .2020 & 30.07 .2020 & \multicolumn{3}{|c|}{ 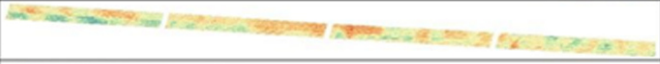 } & 0.096 & 0.543 \\
\hline 18.06 .2020 & 06.08 .2020 & \multicolumn{3}{|c|}{ 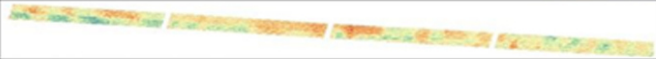 } & 0.107 & 0.581 \\
\hline 18.06 .2020 & 13.08 .2020 & \multicolumn{3}{|c|}{ 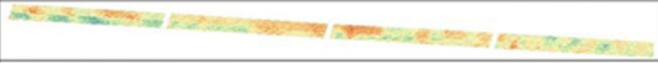 } & 0.121 & 0.606 \\
\hline 18.06 .2020 & 20.08 .2020 & \multicolumn{3}{|c|}{ 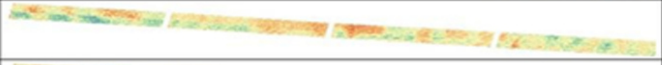 } & 0.133 & 0.624 \\
\hline 18.06 .2020 & 27.08 .2020 & \multicolumn{3}{|c|}{ 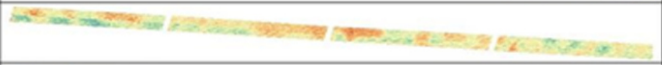 } & 0.140 & 0.634 \\
\hline 18.06 .2020 & 03.09 .2020 & \multicolumn{3}{|c|}{ 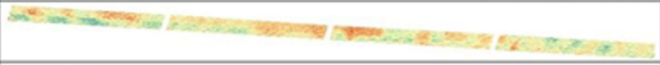 } & 0.147 & 0.643 \\
\hline 18.06 .2020 & 10.09 .2020 & \multicolumn{3}{|c|}{ 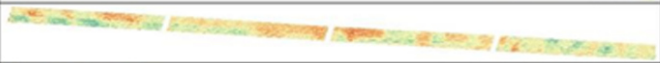 } & 0.153 & 0.633 \\
\hline 18.06 .2020 & 16.09 .2020 & \multicolumn{3}{|c|}{ 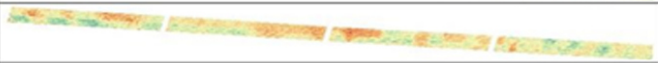 } & 0.158 & 0.661 \\
\hline
\end{tabular}

Fig. 4. Cartograms of the MSAVI2 wheat crops integral during the growing season (from June 18 to September 16, 2020), obtained from unmanned data.

It was found that the spatial-temporal heterogeneity of satellite data is smoothed out on the cartograms of the vegetation indices integrals, as well as on unmanned data, but at the same time a fine spatial structure is preserved on unmanned data. These results allow to conclude that for early forecasting without taking into account the fine spatial structure, it is more preferable to use satellite data, and when assessing the intra-field structure of yield, less reliable results on specific yield can be obtained, but more accurate in the location of plots with higher and lower productivity.

To predict the wheat yield at the beginning of July 2020, linear regression models were calculated, in which the integral values under the MSAVI2 curve at different time periods were used as parameters. The multiple linear model for forecasting wheat from satellite data has the form for 6 variables (determination coefficient 0.72 ):

$$
\mathrm{Y}=-50.73+14.17 \times \text { Imsavi } 2_{1}+5.38 \times \text { Imsavi } 2_{2}-13.18 \times \text { Imsavi } 2_{3}-5.22 \times \text { Imsavi } 2_{4}-
$$

$19.14 \times$ Imsavi $25+23.4 \times$ Imsavi $2_{6}$

A multiple linear model for forecasting wheat based on unmanned data has the form for 2 variables (coefficient of determination 0.49 ):

$$
\mathrm{Y}=14.33-627.23 \times \mathrm{Imsavi}_{2}+429.31 \times \mathrm{Imsavi}_{2}
$$

where Imsavi $2_{\mathrm{i}}$ is the integral value under the MSAVI2 curve at time $\mathrm{i}$; $\mathrm{Y}$ is the yield value, c / ha.

The calculations made it possible to construct a yield cartogram of the study site. Figures 5 6 (I and II) show the spatial distributions of the study site wheat yield, obtained from the model calculations results using unmanned (I) and satellite data (II). 


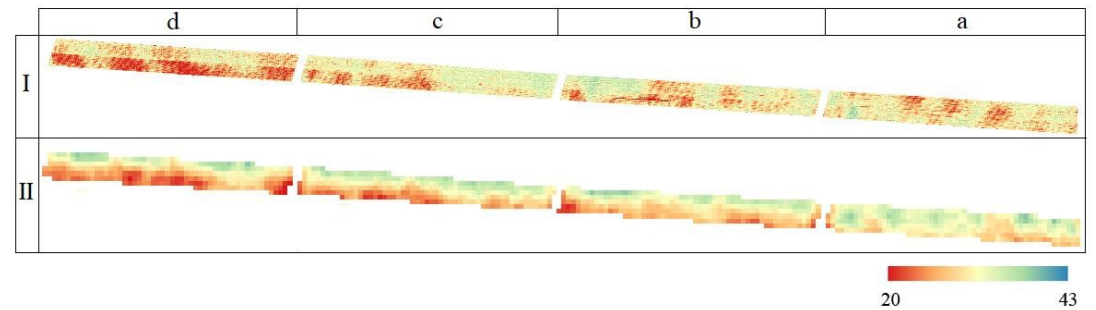

Fig. 5. Maps of the spring wheat yield by fallow (3rd strip), obtained from the results of a model calculation using unmanned data (I) and the results of a model calculation using satellite data (II).

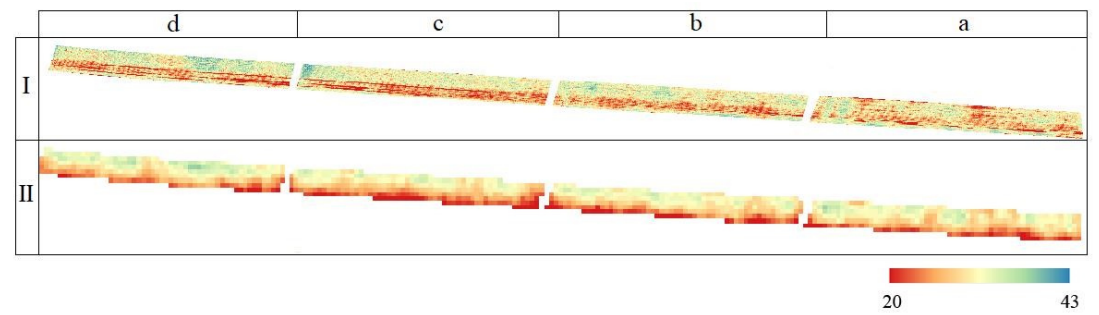

Fig. 6. Maps of the spring wheat yield by corn (5th strip), obtained from the results of a model calculation using unmanned data (I) and the results of a model calculation using satellite data (II).

Comparison of cartograms obtained from the results of model calculations using unmanned (I) and satellite (II) data (Fig. 5, 6) show the similarity of the spatial distribution of yield. The higher spatial resolution of unmanned data $(5 \mathrm{~cm})$ gives a clearer picture of crop heterogeneity, including the presence and absence of fertilized background. In the future, the maps obtained from unmanned data can be successfully applied for differentiated fertilization and selection of soil samples.

The study was carried out with the support of the Krasnoyarsk Regional Science Foundation within the framework of the project: "Development and implementation of a method for early forecasting of grain yield in the Siberian region according to remote sensing data of the Earth".

\section{References}

1. C.F. Jordan, Ecology, Ecology, 50 (1969)

2. A.A. Gitelson, R. Stark, U.Grits, D.Rundquist, Y.Kaufman, D. Derry, International Journal of Remote Sensing, 23 (2002)

3. J. Qi, A. Chehbouni, A.R. Huete, Y.H. Kerr, S. Sorooshian, Remote Sensing of Environment, 48 (1994)

4. G. Dall'Olmo, A.A.Gitelson, D.C. Rundquista, B. Leavitt, T. Barrow, J.C. Holz, Remote Sensing of Environment, 96 (2005) 